\title{
SINGLE-PARTICLE PROPERTIES OF THE EXTENDED HUBBARD MODEL IN THE COMPOSITE OPERATOR METHOD
}

\author{
A. Avella, F. Mancini, M. M. Sánchez \\ Università degli Studi di Salerno - Unità INFM di Salerno \\ Dipartimento di Scienze Fisiche "E. R. Caianiello" \\ 84081 Baronissi (SA), Italy \\ (Received March 9, 1998)
}

\begin{abstract}
Strongly correlated electron systems with planar structure, like high- $T_{c}$ superconductors, are currently under intensive study. In the search for a realistic model to describe the anomalous behaviors of these systems, we have studied the 2D Extended Hubbard model by means of the Composite Operator Method. The local properties, the density of states and the Fermi surface have been calculated as functions of the model parameters. Particular attention has been paid to the role played by the intersite interaction $V$ which drives important changes in the electronic properties of the system.
\end{abstract}

Key words: extended Hubbard model, composite operator method.

PACS number(s): 71.10.Fd, 74.20.-z

\section{INTRODUCTION}

The Hubbard model contains the essence of strong electronic correlations and has been studied as a minimal model for describing a variety of phenomena ranging from metal-insulator transition to itinerant magnetism. Nevertheless, the electronic properties of materials with strongly correlated electrons still present unclarified issues which call for a generalization of the Hubbard Hamiltonian. Many authors have emphasized the importance of considering non-local Coulomb interaction terms in describing doped systems like the cuprate superconductors or the fullerides [1].

The simplest Hamiltonian satisfying these requirements is the Extended Hubbard model, where a nearestneighbor Coulomb interaction term $V$ is added to the original Hubbard Hamiltonian. The inclusion of nonlocal Coulomb interactions substantially modifies the electronic properties of the model. For instance, the charge transfer excitons, which can only be detected by optical spectroscopy at half-filling, attain some charge in doped systems and become visible in direct and inverse photoelectron spectroscopies [2]. Other studies, using an effective extended Hubbard model, support the appearance, upon doping, of the states evenly distributed inside the gap [3]. This suggests that the general features of the spectral properties of the cuprates can be well described by using this effective extended Hubbard Hamiltonian. The extended Hubbard model, with an attractive intersite Coulomb interaction has also been used to mimic some of the experimental features of the cuprates in the superconducting state by means of a BCS treatment [4].

According to this, we have studied the Extended Hubbard model by means of the Composite Operator method $(\mathrm{COM})$ in the static approximation [5]. In this calculation scheme the long-lived excitations of the system are described by an appropriate combination of the standard fermionic field operators. The properties of the new fermionic fields are determined self-consistently by the dynamics. To fix the internal parameters some symmetry requirements, like the Pauli principle and the particlehole symmetry, are imposed. This procedure permits to recover symmetries that are badly violated by other approaches, and thus is expected to provide a better description of strongly correlated systems.

This systematics has been applied to the study of several models, like the $p-d$ model, the 1D and 2D Hubbard models, and the $t-t^{\prime}-U$ model among others [5]. The results obtained for the thermodynamic and magnetic properties are in good agreement with the numerical data. Some anomalous behaviors, experimentally observed in high- $T_{c}$ cuprate superconductors, have also been explained successfully.

In the present work we have studied the effect of the intersite Coulomb interaction term on some properties of the system; namely, the local properties, the Fermi surface, the van Hove singularity and the Mott-Hubbard transition.

\section{METHOD}

The Extended Hubbard model is described by the following Hamiltonian:

$$
\begin{aligned}
H & =\sum_{i j} t_{i j} c^{\dagger}(i) c(j)+U \sum_{i} n_{\uparrow}(i) n_{\downarrow}(i) \\
& +V \sum_{\langle i j\rangle} n(i) n(j)-\mu \sum_{i} n(i),
\end{aligned}
$$

where $c^{\dagger}(i)=\left(c_{\uparrow}^{\dagger}(i), c_{\downarrow}^{\dagger}(i)\right)$ is the electron operator on site $i$ in the spinor notation, $n_{\sigma}(i)$ is the charge-density operator for the spin $\sigma$ and $n(i)$ is the total chargedensity operator. In the hopping matrix 


$$
\begin{aligned}
& t_{i j}=-4 t \alpha_{i j}=-4 t \frac{1}{N} \sum_{k} e^{i \mathbf{k}(i-j)} \alpha(\mathbf{k}), \\
& \alpha(\mathbf{k})=\frac{1}{2}\left(\cos k_{x}+\cos k_{y}\right)
\end{aligned}
$$

only the terms up to the nearest neighbors have been considered; $\alpha_{i j}$ is the projector on the nearest neighbors. The $U$ and $V$ parameters are the onsite and intersite Coulomb repulsions, respectively, and $\mu$ the chemical potential.

The Extended Hubbard model has been analyzed by means of COM in the static approximation, where finite life-time effects are neglected, using the Hubbard doublet as a basic field. The single-particle retarded thermal Green's function is a function of the external parameters $t, U, n$ (filling) and $T$ (temperature) and four internal parameters: $\mu, \Delta, p$ and $\chi_{0}^{\alpha}$. The parameters $\Delta$ and $p$ have already been defined in Ref. [5]. $\chi_{0}^{\alpha}=\sum_{j} \alpha_{i j}\langle n(i) n(j)\rangle$ is the charge susceptibility calculated between nearest neighbor sites. They satisfy the following system of coupled self-consistent equations

$$
\left\{\begin{array}{l}
n=2\left(1-S_{11}-S_{22}\right) \\
\Delta=S_{11}^{\alpha}-S_{22}^{\alpha} \\
S_{12}=0 \\
\chi_{0}^{\alpha}=n^{2}-2 I_{1} I_{2} S_{22}^{-1}\left(I_{1}^{-1}\left(S_{11}^{\alpha}+S_{12}^{\alpha}\right)^{2}\right. \\
\left.\quad+I_{2}^{-1}\left(S_{12}^{\alpha}+S_{22}^{\alpha}\right)^{2}\right)
\end{array}\right.
$$

coming from the existing relations with the elements of the Green's function ( $1^{\text {st }}$ and $2^{\text {nd }}$ equations), the requirement of the Pauli principle at the level of matrix elements ( $3^{r d}$ equation) and the use of the equations of motion $\left(4^{\text {th }}\right.$ equation). $I$ is the renormalization matrix

$$
\begin{aligned}
& I_{1}=1-\frac{n}{2}, \\
& I_{2}=\frac{n}{2} .
\end{aligned}
$$

$S_{\alpha \beta}$ and $S_{\alpha \beta}^{\alpha}$ are the elements of the Green's function calculated on the same site and on nearest-neighbor sites, respectively.

In the framework of COM, the Fourier transform of the single-particle retarded thermal Green's function may be written as:

$$
S(\mathbf{k}, \omega)=\sum_{i=1}^{2} \frac{\sigma^{i}(\mathbf{k})}{\omega-E_{i}(\mathbf{k})}
$$

The changes driven by the presence of the $V$ term can be summarized by giving those in the $m$ matrix

$$
m(\mathbf{k})=m^{0}(\mathbf{k})+V m^{V}(\mathbf{k})
$$

$$
\begin{aligned}
& m_{11}^{V}(\mathbf{k})=4\left(n-\frac{1}{2} \chi_{0}^{\alpha}\right)+4 \alpha(\mathbf{k}) S_{11}^{\alpha}, \\
& m_{12}^{V}(\mathbf{k})=4 \alpha(\mathbf{k}) S_{12}^{\alpha}, \\
& m_{22}^{V}(\mathbf{k})=2 \chi_{0}^{\alpha}+4 \alpha(\mathbf{k}) S_{22}^{\alpha},
\end{aligned}
$$

where $m^{0}(\mathbf{k})$ is the simple Hubbard part, already shown in Ref. [5]. All the other relevant quantities can be calculated from the energy matrix $\varepsilon(\mathbf{k})=m(\mathbf{k}) I^{-1}$; for instance, the energy bands $E_{1,2}(\mathbf{k})$ are the eigenvalues of $\varepsilon(\mathbf{k})$ and the $\sigma^{1,2}(\mathbf{k})$ are simple functions of its elements [5].

\section{RESULTS}

The study of the local properties of the model has shown the following behaviors: near half-filling the chemical potential, the double occupancy and the mobility increase on increasing $V$ and drive a lowering of the total energy of the system (see Figs. 1(a) and 1(b)). Any additional particle has a certain probability to be the nearest neighbor of some others already present in the system leading to an increment of the chemical potential. The cost in energy $U$ of a double occupied site is in part balanced by the energy $V$ of two particles on the nearest neighbor sites. This results in a greater double occupancy. The mobility increases according to the overall tendency towards a charge density instability driven by the $V$ term in agreement with the mean-field result $[6]$.
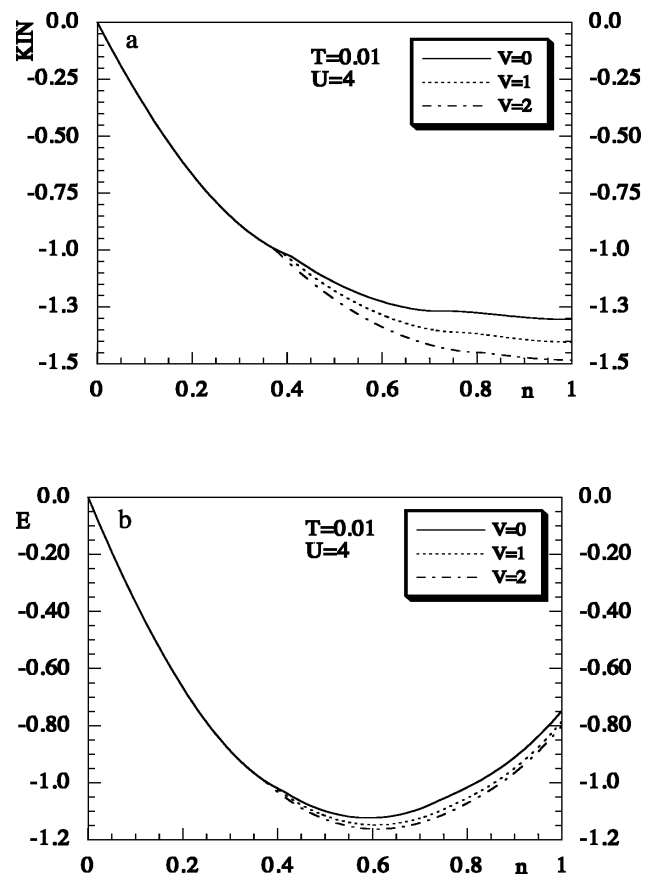

Fig. 1. (a) Kinetic energy for various values of $V$; (b) Total energy for various values of $V$. 

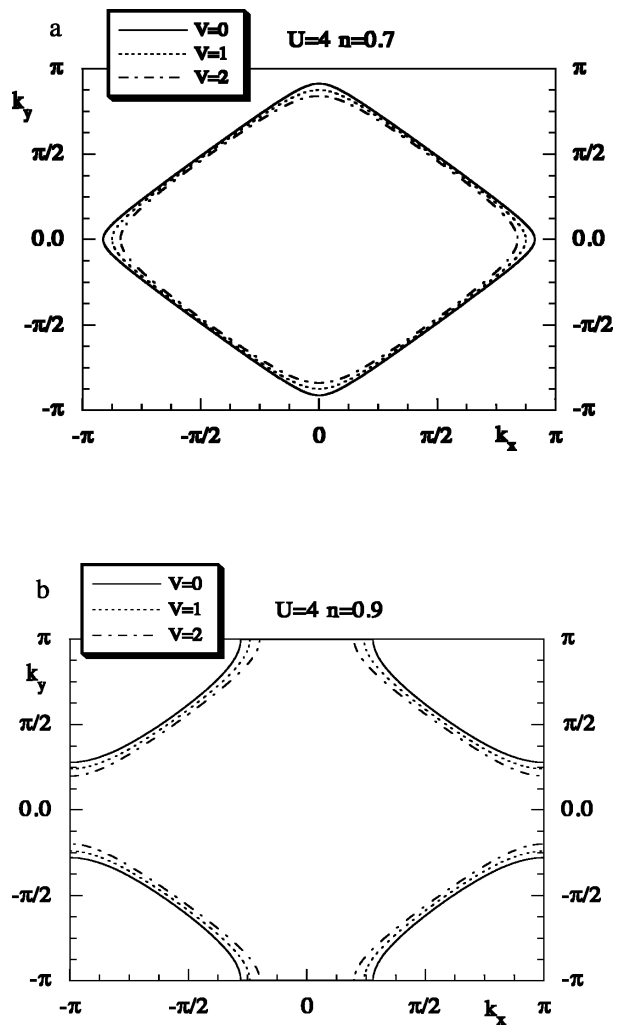

Fig. 2. (a) Fermi surface for various values of $V$ at $n=0.7$; (b) As in (a) at $n=0.9$.
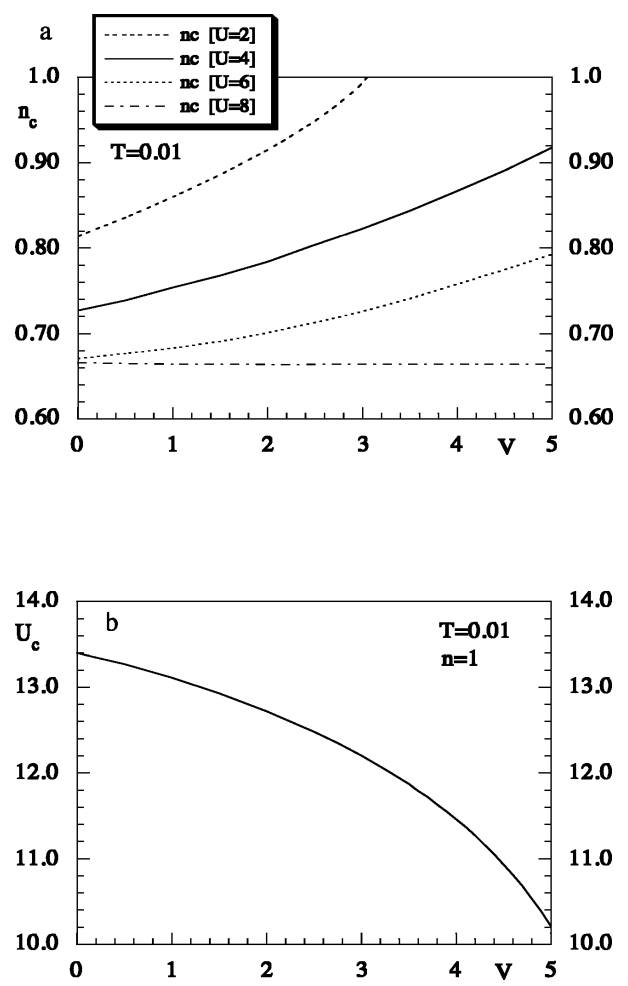

Fig. 3. (a) $n_{c}$ as a function of $V$ for various values of $U$; (b) $U_{c}$ as a function of $V$.
The Fermi surface of the system does not change its overall shape and bending on varying $V$, but, almost rigidly, decreases its volume on increasing $V$ (see Figs. 2(a) and 2(b)). This can be explained as an isotropic increment of the available states in $\mathbf{k}$-space and can be useful to describe quantitatively rather than qualitatively (as the usual Hubbard model does) the ARPES determinations of the Fermi surfaces of the cuprate superconductors [7].

Static susceptibility [8] and specific heat [9] measurements in the cuprate superconductors have shown the presence of well-defined peaks as functions of the filling. This feature, within the framework of the van Hove scenario, is related to the crossing of the Fermi level through a van Hove singularity in the density of states. We have studied the value of the filling $n_{c}$, at which this crossing occurs. The result is shown in Fig. 3(a), as a function of $U$ and $V$. As we can see, $n_{c}$ increases on increasing $V$ until a certain value of $U$ is reached. Above this critical value of $U$ the influence of $V$ is almost null. This is a clear indication that if we would like to explain the cuprates superconductors and their anomalous features by means of models á la Hubbard we need to exploit the intermediate regime for the onsite coupling and the weak regime for the intersite one. Only in this region of the model parameters, $n_{c}$ is in qualitative agreement with the experimental data $[8,9]$.

The physical quantity that mostly feels the intersite Coulomb repulsion $V$ is $U_{c}$, i.e. the critical value of $U$ that marks the Mott-Hubbard transition in the model. As we can see in Fig. 3(b), $U_{c}$ decreases rapidly and becomes of the order of the bandwidth for high values of $V$. This can be understood as the signature of an instability of the system towards a phase with a strong charge ordering.

\section{CONCLUSIONS}

The variety of phenomena present in the strongly correlated electron materials calls for a generalization of the Hubbard model. The inclusion of non-local Coulomb interaction terms is considered important for describing the anomalous behaviors of some doped systems like the cuprate superconductors or the fullerides.

According to this, we have studied the Extended Hubbard Hamiltonian by means of the Composite Operator Method. Using this fully self-consistent treatment we have obtained both the local and the single-particle properties of the model. The former show a tendency towards a charge ordered phase instability. The latter are modified quantitatively, but not qualitatively, with respect to the results obtained with the usual Hubbard Hamiltonian. This could permit to fit more accurately the experimental data for the cuprates superconductors.

The value of the filling at which the Fermi level crosses the van Hove singularity in the density of states has been studied as a function of the coupling constants. A comparison with the experimental data for the magnetic and 
thermodynamical properties of high- $T_{c}$ superconductors suggests an intermediate regime for the onsite coupling and a weak regime for the intersite one as most adequate for the description of these materials.
[1] C. M. Varma, Solid State Commun. 62, 681 (1987); P. B. Littlewood, C. M. Varma, E. Abrahams, Phys. Rev. Lett. 63, 2602 (1989); C. M. Varma, Phys. Rev. Lett. 75, 898 (1995); A. M. Janner et al., Phys. Rev. B 52, 17158 (1995); J. van den Brink et al., Phys. Rev. Lett. 75, 4658 (1995).

[2] J. van den Brink, R. Eder, G. A. Sawatzky, preprint condmat/9704004 (1997).

[3] M. E. Simón, A. A. Aligia, E. R. Gagliano, preprint condmat/9707128 (1997).

[4] J. Ferrer, M. A. González-Alvarez, J. Sánchez-Cañizares, preprint cond-mat/9801109 (1998).

[5] F. Mancini, S. Marra, H. Matsumoto, Physica C 244, 49 (1995); 250, 184 (1995); 252, 361 (1995); F. Mancini, S. Marra, D. Villani, H. Matsumoto, Phys. Lett. A 210, 429 (1996); A. Avella, F. Mancini, M. M. Sánchez, D. Vil- lani, F. D. Buzatu, J. Phys. Stud. 2, (1998); A. Avella, F. Mancini, H. Matsumoto, D. Villani, Physica C 282287, 1757 (1997); 282-287, 1759 (1997).

[6] B. Chattopadhyay, D. M. Gaitonde, preprint condmat/9703213 (1997).

[7] R. S. Markiewicz, preprint cond-mat/9611238 (1996), published in J. Phys. Chem. Solids.

[8] J. B. Torrance et al., Phys. Rev. B 40, 8872 (1989); D. C. Johnston, Phys. Rev. Lett. 62, 957 (1989).

[9] J. W. Loram, K. A. Mirza, W. Y. Liang, J. Osborne, Physica C 162, 498 (1989); J. W. Loram, K. A. Mirza, J. R. Cooper, W. Y. Liang, Phys. Rev. Lett. 71, 1740 (1993); N. Wada, T. Obana, Y. Nakamura, K. Kumagai, Physica B 165-166, 1341 (1990); J. W. Loram, K. A. Mirza, J. M. Wade, J. R. Cooper, W. Y. Liang, Physica C 235-240, 134 (1994). 